Proceedings of International Mathematical Sciences

ISSN:2717-6355, URL: HTTPS://DERGIPARK.ORG.TR/TR/PUB/PIMS

Volume III Issue 2 (2021), PAges 88-97.

DOI: HTTPS://DOI.ORG/10.47086/PIMS.985275

\title{
ON MULTISET MINIMAL STRUCTURE TOPOLOGICAL SPACE
}

\author{
RAKHAL DAS, SUMAN DAS, BINOD CHANDRA TRIPATHY \\ TRIPURA UNIVERSITY, AGARTALA, INDIA
}

\begin{abstract}
In this article we have established the concept of multi-continuity in minimal structure spaces (in short $\mathscr{M}$ space) and the notion of product minimal space in Multiset topological space. Continuity between $\mathscr{M}$-space, generalized Multiset topology and Multiset ideal topological spaces. We have investigated some basic properties of $\mathscr{M}$-continuity in Multiset topological space, such as composition of $\mathscr{M}$-continuous functions, product of $\mathscr{M}$-continuous functions in product Multiset topological space etc.
\end{abstract}

\section{INTRODUCTION}

Cantor's set is not enough for representing the all kind of situations of our real world. In Cantor's set theory, repetition of elements is not allowed. however, there are many situations where repetition of elements plays a vital role. This led the introduction of the theory of the notion of Multisets, which was first studied by Blizard [1] in the year 1989. Thus, a Multiset is a collection of elements in which certain elements may occur more than once and number of times an element occurs is called its multiplicity.

In this article our aim is to study the properties of continuous function on Multiset minimal space and Multiset generalized topological spaces. Minimal stricture space is the minimum restriction for the topology by containing empty set and whole set. Many authors have studied in the direction of Multiset ideal and generalized Multiset topological spaces. This work aim is relating the Multiset ideal, Multiset filter and generalized Multiset topological space

In 1991 Bilzard[2] designed the Multiset theory and further developed the Multiset theory in 1989. Many researchers have defined the Multiset topological spaces; one may refer to New axioms in topological spaces[4], Separation axioms on Multiset topological Space [5], Relations and functions in Multiset context [6]. There after different properties of Multiset topological space, such as compactness studied by Mahanta and Samanta [9], Multiset quasicoincidence studied by Shravan and

2020 Mathematics Subject Classification. 03E70, 54A05, 54A35.

Key words and phrases. Multiset, multiset topology, minimal structure, multiset pre-open set, multiset semi-open set, $\mathscr{M}$-continuity, multiset product space, generalized multiset, ideal.

(C) 2021 Proceedings of International Mathematical Sciences.

Submitted on 20.08.2021, Accepted on 02.01.2022.

Communicated by Ljubiša D. R. KOČİNAC. 
Tripathy Multiset quasiconcidence between Multisets, continuous function on Multisets, generalized closed Multiset $[12,13,14,15]$. Multiset mixed topological space between two Multisets studied by Tripathy and Das [16].

In this article we have established many results between Multiset minimal stricture space and Multiset ideal topological space.

We define different types of continuous function between two multiset generalized topological space, Multiset ideal and generalized topological space. By the results of this article we can study the ideal structure and generalized topological structure at a time, and we can can find the similarity between the topological structures.

This paper is organized in the following way:

Section-1 is the introduction part. In this section, author focused on the previous work and back ground of the research. In the Section-2, author provides some preliminary results and definitions for the article which is necessary for the work. Section-3 is the main section in which we established main results of this article. In Section-4, we analyzed the continuous function from Multiset minimal stricture to generalized Multiset topology and Multiset ideal topological spaces. Section-5 is the conclusion section in which the future plane and the application field have been analyzed by the author.

\section{Preliminaries and Definitions}

In this section, we provide some basic definitions and notations those will be used throughout this article.

A Multiset (M-set) with domain set $X$, in which no element occurs more than $m$ times is denoted by $[X]^{m}$. The count function $C_{m}$ on $X$ represents the repetition of an element, denoted by $C_{m}(x)$, for $x \in X$. When $C_{m}(x)=1$, for all $x \in X$, then the Multiset becomes a Cantor's set.

Thought the articles we shall use the definition of Multiset mixed topological space (Shravan and Tripathy [13]) and ultra-Separation Axioms in Generalized Topological Space (Powar and Rajak [10]) for the union, intersection, compliment, support set, empty set, equality of M-sets, partial whole sub-M-sets etc.

Definition 2.1. A domain $X$ is defined as a set of elements from which M-sets are constructed. The M-set space $[X]^{m}$ is the set of all M-set whose elements are in $X$ such that no element in the M-set occurs more than $m$ times. The set $[X]^{\infty}$ is the set of all M-sets over a domain $X$ such that there is no limit on the number of occurrences of an element in an M-set.

Let $P, N \in[X]^{m}$. Then, the following relations between M-sets are defined:

(1) $P$ is a sub-M-set of $N$ denoted by $P \subseteq N$, if $C_{P}(x) \leq C_{N}(x)$ for all $x \in X$.

(2) $P=N$ if $P \subseteq N$ and $N \subseteq P$.

(3) $P$ is a proper sub-M-set of $N$ denoted by $P \subset N$, if $C_{P}(x) \leq C_{N}(x)$, for all $x \in X$ and there exists at least one element $x \in X$ such that $C_{P}(x)<C_{N}(x)$. 
(4) $Q=P \cup N$, if $C_{Q}(x)=\max \left\{C_{P}(x), C_{N}(x)\right\}$, for all $x \in X$.

(5) $Q=P \cap N$, if $C_{Q}(x)=\min \left\{C_{P}(x), C_{N}(x)\right\}$, for all $x \in X$.

(6) Addition of $P$ and $N$ is also a new M-set $Q=P \bigoplus N$ such that $C_{Q}(x)=$ $\min \left\{C_{P}(x)+C_{N}(x), m\right\}$, for all $x \in X$.

(7) Subtraction of $P$ and $N$ results is also an M-set $Q=P \ominus N$ such that $C_{Q}(x)=\max \left\{C_{P}(x)-C_{N}(x), 0\right\}$, for all $x \in X$, where $\bigoplus$ and $\ominus$ represent M-set addition and M-set subtraction, respectively.

(8) An M-set $P$ is empty if $C_{P}(x)=0$, for all $x \in X$.

(9) The support set of $P$ denoted by $P^{*}$ is a subset of $X$ and $P^{*}=\{x \in X$ : $\left.C_{P}(x)>0\right\}$; that is, $P^{*}$ is an ordinary set and it is also called root set.

(10) The cardinality of an M-set $P$ drawn from a set $X$ is $\operatorname{Card}(P)=\sum_{x \in X} C_{P}(x)$.

(11) $P$ and $N$ are said to be equivalent if and only if $\operatorname{Card}(P)=\operatorname{Card}(N)$.

Definition 2.2. Let $M \in[X]^{m}$ and $N \subseteq M$. Then, the complement $N^{c}$ of $N$ in $[X]^{m}$ is an element of $[X]^{m}$ such that $N^{c}=M-N$.

Definition 2.3. Let $M \subseteq[X]^{m}$ and $P^{*}(M)$. Then $\tau$ is called a Multiset topology of $M$ if satisfies the following properties,

1. The whole M-set $M$ and the empty M-set $\emptyset$ are in $\tau$.

2. The M-set union of elements of any sub-collection of $\tau$ is in $\tau$.

3. The M-set intersection of the elements of any finite sub-collection of $\tau$ is in $\tau$.

Then, the pair $(M, \tau)$ is called an Multiset topological space ( $M$-topological space). The elements of $\tau$ are called open M-sets. The complement of an open M-set in $(M, \tau)$ is said to be closed M-set.

Definition 2.4. Given a sub-M-set $N$ of $M$-topological space. Then, the interior of $N$ is denoted by $\operatorname{int}(N)$ and is defined as the M-set union of all open M-sets contained in $N$, i.e., $C_{\text {int }(N)}(x)=\max \left\{C_{G}(x): G \subseteq N\right\}$.

Definition 2.5. Given a sub-M-set $A$ of an $M$-topological space $(M, \tau)$. Then, the closure of $A$ is defined as the M-set intersection of all closed M-sets containing $A$ and is denoted by $\operatorname{cl}(A)$, i.e., $C_{c l(A)}(x)=\min \left\{C_{K}(x): A \subseteq K\right\}$.

Definition 2.6. Let $(M, \tau)$ be an $M$-topological space, and $M_{1}$ is a sub-M-set of $M$. The collection $\tau_{N}=\left\{U^{\prime}=U \cap M_{1}: U \in \tau\right\}$ is an $M$-topology on $M_{1}$, called the subspace $M$-topology. With this $M$-topology, $M_{1}$ is called a subspace of $M$ and its open M-sets consisting of all M-set intersections of open M-sets of $M$ with $M_{1}$.

Definition 2.7. A non-empty collection $I$ of sub-M-sets of a non-empty M-set $M$ is said to be an M-set ideal on $M$, if it satisfies the following conditions: 
(i). $N_{1} \in I$ and $N_{2} \subseteq N_{1}$ with $C_{N_{2}}(x) \leq C_{N_{1}}(x)$, for all $x \in X \rightarrow N_{2} \in I$.

(ii). $N_{1} \in I, N_{2} \in I \rightarrow N_{1} \cup N_{2} \in I$. The M-set ideal is abbreviated as $M$-ideal. The triplet $(M, \tau, I)$ is called Multiset ideal topological space with the ideal $I$ and Multiset topology $\tau$.

Definition 2.8. Let $[X]^{w}$ be a space of M-sets. A Multipoint is a M-set $M$ in $X$ such that $C_{M}(x)=\left\{\begin{array}{l}k, \text { for } x \in M ; \\ 0, \text { otherwise }\end{array}\right.$

where $k \in\{1,2,3, \ldots \ldots . w\}$ and $C_{M}(x)$ is the multiplicity of $x$ in $X$.

A multipoint, denoted by $\{k / x\}$ is a subset of a M-set $M$ or $\{k / x\} \in M$ if $0 \leq$ $k \leq C_{M}(x)$ and Singleton sub-M-set if $k=C_{M}(x)$, for all $x \in X$.

Let $(M, \tau)$ be a $M$-topological space and $I$ be an $M$-ideal on $M$. Let $N$ be a sub-M-set of $M$. Then, the local function denoted by $N^{*}(I, \tau)$ is defined by, $N^{*}(I, \tau)=\left\{m_{i} / x_{i} \in M: C_{U}\left(x_{j}\right)-C_{N^{c}}\left(x_{j}\right)>C_{I}\left(x_{j}\right), I \in I\right.$, for all $U \in N_{q}\left(m_{i} / x_{i}\right)$ and at least one $x_{j} \in X$ \}, where $N_{q}\left(m_{i} / x_{i}\right)$ is the set of $q-n b h d$ of $m_{i} / x_{i}$. We will write $N^{*}(I)$ or $N^{*}$ in place of $N^{*}(I, \tau)$.

Definition 2.9. Let $M$ be any non-empty M-set and $\tau$ be the collection of subsets of the M-set $M$. the pair $(M, \tau)$ is said to be a generalized M-set topological space if the following property holds

1. $M, \emptyset \in \tau$.

2. If $H, G \in \tau$ then $H \cap G \in \tau$.

3. If $u_{i \in \Lambda} \in \tau$ then $\cup_{i \in \Lambda} u_{i} \in \tau$.

Note 2.1. The generalized M-set topological space is the generalized form of M-set topology. Sometimes, we denote generalized M-set topology by $(M(N), \tau)$, where $\cup_{i \in \Lambda} u_{i}=N$.

\section{Main Results.}

In this section we established a topology from the M-set minimal structure, and study different properties on multi-continuity between the different types of M-set topological space and M-set minimal space.

Definition 3.1. A family $\mathscr{M} \subseteq 2^{X}(\mathscr{P}(X)$ is said to be M-set minimal structure on $X$ if $\emptyset, X \in \mathscr{M}$.

In this case $(M, \mathscr{M})$ is called a M-set minimal space. Throughout this paper $(X, \mathscr{M})$ means M-set minimal space. The M-set minimal space is abbreviated as $M$-minimal space.

Definition 3.2. A $M$-minimal space is called an M-set minimal topological space if it satisfy the properties of finite intersection and arbitrary union property. 
Example 3.1. Let $M$ be a nonempty M-set on $[X]^{w}$. Then, the filter $\mathscr{F}$ and the ideal do not form a M-set minimal structure on $X$. Since, $\mathscr{F}$ does not contain emptyset $\emptyset$ and ideal does not contain the whole set $M$.

Definition 3.3. A set $A \in P^{*}(X)$ is said to be a $\mathscr{M}$-open set if for $A \in \mathscr{M}$, $B \in P^{*}(X)$ is a $\mathscr{M}$-closed set if $M \ominus B \in \mathscr{M}$. We get

$\mathscr{M}-\operatorname{int}(A)=\cup\{u: u \subseteq A, u \in \mathscr{M}\}$.

$\mathscr{M}-\operatorname{cl}(A)=\cap\{F: A \subseteq F, M \ominus F \in \mathscr{M}\}$.

In view of above definition we formulate the following proposition:

\section{Proposition 3.1.}

1. $\mathscr{M}-\operatorname{int}(A)$ is the largest $\mathscr{M}$-open M-set contained in $A$.

2. $\mathscr{M}-\operatorname{cl}(A)$ is the smallest $\mathscr{M}$-closed M-set containing $A$.

Definition 3.4. Let $(M, \mathscr{M})$ be a M-set $\mathscr{M}$-space on $[X]^{w}$, and $N$ be a sub-Mset of $M$. We define the following

(i) A $\mathscr{M}$-semi-open M-set if $N \subseteq C l(\operatorname{int}(N))$ with $C_{N}(x) \leq C_{c l(\operatorname{int}(N))}(x)$, for all $x \in X$;

(ii) A $\mathscr{M}$-semi-closed M-set if $\operatorname{int}(\operatorname{cl}(N)) \subseteq N$ with $C_{\text {int }(\operatorname{cl}(N))}(x) \leq C_{N}(x)$, for all $x \in X$;

(iii) A $\mathscr{M}$-semi-pre-open M-set if $N \subseteq C l(\operatorname{int}(\operatorname{cl}(N)))$ with $C_{N}(x) \leq C_{c l(\operatorname{int}(\operatorname{cl}(N)))}(x)$, for all $x \in X$;

(iv) A $\mathscr{M}$-semi-pre closed M-set if $\operatorname{int}(\operatorname{cl}(\operatorname{int}(N))) \subseteq N$ with $C_{\text {int }(\operatorname{cl}(\operatorname{int}(N)))}(x) \leq$ $C_{N}(x)$, for all $x \in X$;

(v) A $\mathscr{M}$-pre-open M-set if $N \subseteq \operatorname{int}(\operatorname{cl}(N))$ with $C_{N}(x) \leq C_{i n t(c l(N)))}(x)$, for all $x \in X$.

Theorem 3.1. Let $(M, \mathscr{M})$ be a $M$-minimal space. Then, for $N, K \in P^{*}(X)$,

1. $\mathscr{M}-\operatorname{int}(N) \subseteq N$ and $\mathscr{M}-\operatorname{int}(N)=N$ iff $N$ is an $\mathscr{M}$-open M-set.

2. $\mathscr{M}-\operatorname{cl}(N) \subseteq N$ iff $N$ is an $\mathscr{M}$-closed M-set.

3. $\mathscr{M}-\operatorname{int}(N) \subseteq \mathscr{M}-\operatorname{int}(K)$ and $\mathscr{M}-\operatorname{cl}(N) \subseteq \mathscr{M}-\operatorname{cl}(K)$ if $N \subseteq K$.

4. $\mathscr{M}-\operatorname{int}(N \cap K) \subseteq(\mathscr{M}-\operatorname{int}(N)) \cap(\mathscr{M}-\operatorname{int}(K))$ and $(\mathscr{M}-\operatorname{int}(N)) \cup(\mathscr{M}-$ $\operatorname{int}(K)) \cup \mathscr{M}-\operatorname{int}(N \cup K)$.

5. $\mathscr{M}-\operatorname{cl}(N \cup K) \subseteq(\mathscr{M}-\operatorname{cl}(N)) \cup(\mathscr{M}-\operatorname{cl}(K))$ and $\mathscr{M}-\operatorname{cl}(N \cap K) \subseteq$ $(\mathscr{M}-\operatorname{cl}(N)) \cup(\mathscr{M}-\operatorname{cl}(\bar{K}))$.

6. $\mathscr{M}-\operatorname{int}(\mathscr{M}-\operatorname{int}(N))=\mathscr{M}-\operatorname{int}(N)$ and $\mathscr{M}-\operatorname{cl}(\mathscr{M}-\operatorname{cl}(N))=\mathscr{M}-\operatorname{cl}(N)$.

7. $x \in \mathscr{M}-c l(N)$ if and only if every $\mathscr{M}$-open M-set $U$ containing $x$ such that $U \cap N \neq \emptyset$. 
8. $(M \ominus \mathscr{M}-\operatorname{cl}(N))=\mathscr{M}-i n t(M \ominus N)$ and $(M \ominus \mathscr{M}-i n t(N))=\mathscr{M}-C l(M \ominus N)$.

Proof (1) $\mathscr{M}-\operatorname{int}(N)$ is the largest open M-set contained in $N$. So the proof is clear. Now, let us consider $N$ is $\mathscr{M}$-open M-set. Then, $N \subseteq \mathscr{M}-\operatorname{int}(N)$. Therefore, $N=\mathscr{M}-\operatorname{int}(N)$.

Let us consider $N=\mathscr{M}-\operatorname{int}(N)$. Again $\mathscr{M}-\operatorname{int}(N)$ is the largest open M-set contained in $N$. Hence, $N$ is $\mathscr{M}$-open M-set.

(2). Let $\mathscr{M}-\operatorname{cl}(N)$ be a small closed M-set containing $N$. Therefore, $N \subseteq$ $\mathscr{M}-\operatorname{cl}(N)$. Again, $N$ is $\mathscr{M}$-closed M-set. This implies, the closure of all multipoint of $N$ contained in $N$ with $C_{c l(N)}(x) \leq C_{N}(x)$, for all $x \in N$. Hence the theorem proved.

(3) From the definition we have $\mathscr{M}-\operatorname{int}(N) \subseteq N$ and $\mathscr{M}-\operatorname{int}(K) \subseteq K$ for any M-set $N, K \subseteq M$. Given that $N \subseteq K$ this implies, $\mathscr{M}-\operatorname{int}(N) \subseteq N \subseteq K$ So, $\mathscr{M}-\operatorname{int}(N) \subseteq K$. By the definition of M-set interior point set is the largest open M-set of the M-set contained in the M-set. Hence, $\mathscr{M}-\operatorname{int}(K)$ is the largest open M-set in $K$. Again, $\mathscr{M}-\operatorname{int}(N)$ is an open M-set contained in $K$. So the only possible case $\mathscr{M}-\operatorname{int}(N) \subseteq \mathscr{M}-\operatorname{int}(K)$.

(4) For any two M-sets $N$ and $K$, we have $N \cap K \subseteq N$ and $N \cap K \subseteq K$. Using the above results we have, $\mathscr{M}-\operatorname{int}(N \cap K) \subseteq \mathscr{M}-\operatorname{int}(N)$ and $\mathscr{M}-\operatorname{int}(N \cap K) \subseteq$ $\mathscr{M}-\operatorname{int}(K)$. This implies, $\mathscr{M}-\operatorname{int}(N \cap K) \subseteq(\mathscr{M}-\operatorname{int}(N)) \cap(\mathscr{M}-\operatorname{int}(K))$.

Again, for any two M-set $N$ and $K$, we have $N \subseteq N \cup K$ and $K \subseteq N \cup K$. This implies, $\mathscr{M}-\operatorname{int}(N) \subseteq \mathscr{M}-\operatorname{int}(N \cup K)$ and $\overline{\mathscr{M}}-\operatorname{int}(K) \subseteq \mathscr{M}-\operatorname{int}(N \cup K)$. Hence, $(\mathscr{M}-\operatorname{int}(N)) \cup(\mathscr{M}-\operatorname{int}(K)) \subseteq \mathscr{M}-\operatorname{int}(N \cup K)$.

(5) We have, $N \subseteq(m-c l(N)), K \subseteq(\mathscr{M}-c l(K))$. Therefore, $N \cup K \subseteq(\mathscr{M}-$ $\operatorname{cl}(N)) \cup(\mathscr{M}-\operatorname{cl}(K))$ and so $\mathscr{M}-\operatorname{cl}(N \cup K) \subseteq \mathscr{M}-\operatorname{cl}((\mathscr{M}-\operatorname{cl}(N)) \cup(\mathscr{M}-\operatorname{cl}(K)))=$ $(\mathscr{M}-\operatorname{cl}(N)) \cup \mathscr{M}-\operatorname{cl}(K))$.

Hence, $m-\operatorname{cl}(N \cup K) \subseteq(m-\operatorname{cl}(N)) \cup \mathscr{M}-c l(K))$ as union of two $\mathscr{M}$-closed M-sets is also a $\mathscr{M}$-closed M-set and $\mathscr{M}$-closure of a $\mathscr{M}$-closed M-set is also a $\mathscr{M}$-closed M-set.

Similarly, for the $\mathscr{M}-c l(N \cap K) \subseteq(\mathscr{M}-\operatorname{cl}(N)) \cap(\mathscr{M}-\operatorname{cl}(K))$.

For (6), (7) and (8), the results are holds using the definition and above proofs.

Lemma 3.1. A sub-M-set $N$ in a M-set minimal space $(M, \mathscr{M})$ is said to be $\mathscr{M}$-semi-pre-closed M-set if and only if $N=\operatorname{spcl}(N)$.

Definition 3.5. A sub-M-set $N$ of a M-set topological space $(M, \tau)$ is called as (1) M-set generalized semi-closed (briefly $m g s$-closed) set if $m s C l(N) \subseteq U$ whenever $N \subseteq U$ and $U$ is open M-set in generalized $\mathscr{M}$ - space.

(2) M-set generalized minimal semi-pre closed (briefly $\mathscr{M}-m g s p$-closed) set if $\operatorname{spcl}(N) \subseteq U$ whenever $N \subseteq U$ and $U$ is $\mathscr{M}$-open M-set in generalized $M$ space. 
Definition 3.6. Let $\left(M_{1}, \tau_{1}\right)$ and $\left(M_{2}, \tau_{2}\right)$ be two M-set minimal spaces. Then, a function $f: M_{1} \rightarrow M_{2}$ is called as

(i) multi-semi-continuous if the inverse image of each $\mathscr{M}$-open M-set of $M_{2}$ is $\mathscr{M}$-semi-open M-set in $M_{1}$.

(ii) multi minimal semi pre-continuous if the inverse image of each $m$-open M-set of $M_{2}$ is $m$-semi-pre open M-set in $M_{1}$.

(iii) multiset minimal $g$-continuous if the inverse image of each $m$-open M-set of $M_{2}$ is $\mathscr{M}-m g$-open M-set in $M_{1}$.

(iv) multiset minimal $g p$-continuous if the inverse image of each $m$-open M-set of $M_{2}$ is $\mathscr{M}-m g p$-open M-set in $M_{1}$.

(v) multiset minimal $g s p$-continuous if the inverse image of each $m$-open M-set of $M_{2}$ is $\mathscr{M}-g s p$-open M-set in $M_{1}$.

(vi) multiset minimal pre-continuous if the inverse image of each $m$-open M-set of $M_{2}$ is $m$-pre open M-set in $M_{1}$.

Definition 3.7. Let $(M, I, \tau)$ be an M-set ideal topological space on $[X]^{w}$. A subset $A$ of M-set ideal space is said to be pre-I-open M-set if $A \subseteq \operatorname{int}\left(\operatorname{cl}\left(A^{*}\right)\right)$ with $C_{A}(x) \leq C_{\text {int }\left(c l\left(A^{*}\right)\right)}(x)$ for all $x \in X$. The compliment of pre-I-open M-set is called pre-I-closed M-set.

Definition 3.8. Let $(M, I, \tau)$ be an M-set ideal topological space in $[X]^{W}$. A sub $M$-set $A$ of $M$ is called a semi-I-open M-set if $A \subseteq \operatorname{cl}\left(\operatorname{int}\left(A^{*}\right)\right)$ with $C_{A}(x) \leq$ $C_{c l\left(i n t\left(A^{*}\right)\right)}(x)$, for all $x \in X$. The complement of a semi-open $M$-set is called a semi $-I-$ closed $M$-set.

Proposition 3.2. Let $\left(M_{1}, \tau_{1}\right)$ and $\left(M_{2}, \tau_{2}\right)$ be two M-set minimal spaces on $M$. A function $f: M_{1} \rightarrow M_{2}$ be a bijective mapping and $\left\{U_{i}: i \in \Delta\right\}$ be a family of $\mathscr{M}$-open M-sets of $M_{2}$. Then, we have

(i) $f^{-1}\left(\cup_{i \in \Delta} U_{i}\right)=\cup_{i \in \Delta} f^{-1}\left(U_{i}\right)$.

(ii) $f^{-1}\left(\cap_{i \in \Delta} U_{i}\right)=\cap_{i \in \Delta} f^{-1}\left(U_{i}\right)$.

Lemma 3.2. Every M-set topological space is a M-set minimal space, but the converse is not necessary.

Lemma 3.2. Every M-set minimal space is a generalized M-set minimal space, but the converse is not necessary. 


\section{Properties of $\mathscr{M}-m s g p$ Generalized Multiset and IDEAL-CONTINUOUS FunCTIONS}

We state the following two results without proof, which follow on using standard theory.

Theorem 4.1. Let $f:\left(M_{1}, \tau_{1}\right) \rightarrow\left(M_{2}, \tau_{2}\right)$ and $g:\left(M_{2}, \tau_{2}\right) \rightarrow\left(M_{3}, \tau_{3}\right)$ be any two maps. Then, $g \circ f$ is $\mathscr{M}$-continuous if $g$ is $\mathscr{M}$-continuous and $f$ is $\mathscr{M}$ continuous.

Proof: The proof is so easy, so omitted.

Proposition 4.1. Let $\left(M_{1}, \tau\right)$ be M-set minimal space, and $M_{2} \subseteq M_{1}$. Then, $\left(M_{2}, \tau \cap M_{2}\right)$ will be an M-set minimal structure. Further, for $M_{2} \subseteq M_{1},\left(M_{2}, \tau \cap\right.$ $\left.M_{2}\right)$ is a weaker M-set minimal structure space.

Definition 4.1. Let $\left(M_{1}, \tau_{1}\right)$ and $\left(M_{2}, \tau_{2}\right)$ be two M-set minimal spaces. A function $f: M_{1} \rightarrow M_{2}$ is called $\mathscr{M} m g s p$-continuous if $f^{-1}(N)$ if $\mathscr{M}-m g s p$-closed in $M_{1}$ for every closed M-set $N$ of $M_{2}$.

\section{Lemma 4.1.}

1. Every $g$-continuous function is $\mathscr{M}-m g s p$-continuous function.

2. Every $\mathscr{M}-$ pre-continuous function is generalized semi-continuous function.

Theorem 4.2. Let $\left(M_{1}, \tau_{1}\right)$ be a M-set minimal space, and $\left(M_{2}, \tau_{2}\right)$ be a generalized M-set topology. If the bijective function $f: M_{1} \rightarrow M_{2}$ is $\mathscr{M}$-semi-pre continuous and $\mathscr{M}$-open M-set, then $f$ is $\mathscr{M}$ - pre-continious.

Proof: Let $N$ be $\mathscr{M}$-closed in $M_{2}$ and let $f^{-1}(N) \subseteq K$, where $K$ is $\mathscr{M}$-open set in $M_{1}$. Clearly, $N \subseteq f(K)$. Since $f(K)$ is open M-set in $M_{2}$ as $f$ is open, and as $N$ is $\mathscr{M}$-closed in $M_{2}$, then $\mathscr{M}-\operatorname{spCl}(N) \subseteq f(K)$ and thus $f^{-1}(\operatorname{sp} C l(N)) \subseteq K$. Since $f$ is bijective and $\mathscr{M}-m s p C l(N)$ is a $\mathscr{M}$ semi-pre closed M-set, then $f^{-1}(\operatorname{spCl}(N))$ is $\mathscr{M}$-semi-pre closed M-set in $M_{1}$. Thus, $\operatorname{mspCl}\left(f^{-1}(N)\right) \subseteq \operatorname{spCl}\left(f^{-1}(\operatorname{msp} C l(N))\right)$ $=f^{-1}(m s p C l(N)) \subseteq K$. So, $f^{-1}(N)$ is $m g s p$-closed set and $f$ is $\mathscr{M}$--pre-continious.

Theorem 4.3. Let $f: M_{1} \rightarrow M_{2}$ be a pre- $\mathscr{M}-m g s p$-continuous and $g: M_{2} \rightarrow$ $M_{3}$ is $\mathscr{M}$-semi-pre-continuous. Then, their composition $g \circ f$ is $\mathscr{M} m g s p$-continuous. Proof: The proof is straight forward, so omitted.

Definition 4.2. A function $f: M_{1} \rightarrow M_{2}$ is called strongly $\mathscr{M}-m g s p$ continuous if the inverse image of every $\mathscr{M}$-open M-set of $M_{2}$ is $\mathscr{M}$-open M-set in $M_{1}$.

Lemma 4.2. every strongly $\mathscr{M}-m g s p$-continuous function is an $\mathscr{M}$-continuous.

Definition 4.3. A function $f: M_{1} \rightarrow M_{2}$ is called strongly $\mathscr{M}$-continuous if the inverse image of every sub-M-set in $M_{2}$ is $\mathscr{M}-c l-$ open in $M_{1}$. 
On the basis of the above definition we give the following results:

Theorem 4.4. If the function $f: M_{1} \rightarrow M_{2}$ is strongly $\mathscr{M}$-continuous, then $f$ is strongly $\mathscr{M}-m g s p$-continuous.

Theorem 4.5 If $f: M_{1} \rightarrow M_{2}$ be an $m-I-g n$-continuous function from an M-set ideal topological space to another M-set minimal space, then the following are equivalent.

1. for every local function $A^{*}$ in $M_{1}$ there exist an M-set local function $f\left(A^{*}\right)$ in $M_{2}$.

2. For the M-set ideal $I$ there exist an ideal $f(I)$ in $M_{2}$.

3. For every $m g s p$-closed (Open) set in $M_{2}$ there exist an multiset-semi-pre-ideal closed $(\mathscr{M}$-open $)$ set in $M_{1}$.

Theorem 4.6. If the function $f: M_{1} \rightarrow M_{2}$ be $m-I$-continuous and the function $g: M_{2} \rightarrow M_{3}$ be $\mathscr{M}$-continuous, then $g \circ f: M_{1} \rightarrow M_{3}$ is $\mathscr{M}$-continuous.

Proof: Let $N$ be an $\mathscr{M}$-open M-set in $M_{3}$. Since, $g$ is $\mathscr{M}$-continuous, so $g^{-1}(N)$ is an $m-I$-semi-pre-open set in $M_{2}$. Again, $f$ is an $m-I$-continuous. Hence, $f^{-1}\left(g^{-1}(N)\right)$ is an $m-I$-open M-set in $M_{1}$. But, $f^{-1}\left(g^{-1}(N)\right)=(g \circ f)^{-1}(N)$. So $g \circ f$ is an $\mathscr{M}$-continuous.

\section{Conclusion}

In this article, we introduced the notion of M-set minimal space and M-set minimal topological spaces. Besides, we defined different types of M-set minimal continuous function between two M-set minimal space. M-set minimal continuous functions can help to study the structure of M-set minimal spaces. The common property between two M-set minimal structure can be analze by the continious functions. Many interesting results can established between M-set ideal, generalized M-set topological and M-set minimal structure spaces. It is hoped that, in future many new investigations can be done in this direction.

Conflict of Interest: The authors declare that there is no conflict of interest.

Acknowledgement: The authors thank the reviewers for their comments those improved the presentation of the article.

\section{REFERENCES}

[1] W.D. Blizard, Multiset Theory. Notre Dame Jour. Logic, 30 (1989) 36-65.

[2] W.D. Blizard, The development of multiset theory. Modern Logic. 1 (1991) 319-352.

[3] A. Csaszar, Generalized topology, generalized continuity. Acta Math. Hungar., 96(4) (2002) 351-357.

[4] V. Dembre, New axioms in topological spaces. Int. Jour. Comp. Appl. Tech. and Research, 7(3) (2018) 109-113. 
[5] S. El-Sheikh, R. Omar, and M. Raafat, Separation axioms on multiset topological Space. jour. new theory, 7 (2015) 11-21.

[6] K.P. Girish, and S.J. John, Relations and functions in multiset context. Inf. Sci., 179(6) (2009) 758-768.

[7] K.P. Girish, and S.J. John, Multiset topologies induced by multiset relations. Information Sciences, 188 (2012) 298-313.

[8] A. Kanibir, and I.L. Reilly, Generalized continuity for multifunctions. Acta 117 Math. Hungar., 122(3) (2009) 283-292.

[9] S. Mahanta, and S.K Samanta, Compactness in Multiset Topology. Inter. Jour. Math. Trends and Tech. 47(4) (2017) 275-288.

[10] P.L. Powar, and K. Rajak, Ultra-Separation Axioms in Generalized Topological Space. Inte. Jour. Electro Comput. World Know. Inter. 1(4) (2011) 34-40.

[11] K. Shravan, and B.C. Tripathy, Generalised closed sets in multiset topological spaces. Proyecciones Jour. Math., 37(2) (2018) 223-237.

[12] K. Shravan, and B.C. Tripathy, Multiset ideal topological spaces and local functions. Proyecciones Jour. Math., 37(4) (2018) 699-711.

[13] Shravan, K., Tripathy, B.C.: Multiset mixed topological space. Soft Comput, 23 (2019) 98019805.

[14] K. Shravan, and B.C. Tripathy, Multiset ideal topological spaces and Kuratowski closure operator, Bull. Univ. Transilvania Brasov, Series III: Mathematics, Informatics, Physics, 13(62)(1) (2020) 273-284.

[15] K. Shravan, and B.C. Tripathy, Metrizability of multiset topological spaces, Bull. Univ. Transilvania Brasov, Series III: Mathematics, Informatics, Physics, 13(62)(2) (2020) 683-696.

[16] B.C. Tripathy, and G.C. Ray, G.C. Mixed fuzzy ideal topological spaces. Applied Mathematics and Computations; 220 (2013) 602-607.

[17] B.C. Tripathy, and G.C. Ray, G.C. On $\delta$-continuity in mixed fuzzy topological spaces. Boletim da Sociedade Paranaense de Matemática, 32(2) (2014) 175-187).

[18] B.C. Tripathy, and R. Das, G.C. Multiset Mixed Topological Space. Transactions of A. Razmadze Mathematical Institute, (Accepted)

[19] B.C. Tripathy, and G.C. Ray, G.C. Weakly continuous functions on mixed fuzzy topological spaces. Acta Scientiarum. Technology, 36(2) (2014) 331-335.

[20] B.C. Tripathy, and G.C. Ray, G.C. Fuzzy $\delta$ - I-continuity in mixed fuzzy ideal topological spaces. Journal of Applied Analysis, 24(2) (2018) 233-239.

RAKHAL DAS,

Tripura University, Agartala, India

Email address: rakhaldas95@gmail.com

Suman Das,

Tripura University, Agartala, India

Email address: sumandas18842@gmail.com

Binod Chandra Tripathy,

Tripura University, Agartala, India

Email address: tripathybc@gmail.com 